\title{
PROPUESTA DE UN PLAN DE MANEJO DE RESIDUOS SÓLIDOS PELIGROSOS Y NO PELIGROSOS PARA UNA EMPRESA DE MANUFACTURA DE ABRASIVOS
}

\author{
ROPOSAL OF A PLAN OF HAZARDOUS SOLID WASTE MANAGEMENT AND NON- \\ HAZARDOUS FOR ABRASIVE MANUFACTURING COMPANY
}

\author{
${ }^{1}$ Desy Bravo C., ${ }^{2}$ Yenny Galarza O., ${ }^{3}$ Wilfredo Baldeón Q. y ${ }^{4}$.Mary Flor Césare C.
}

\begin{abstract}
Resumen
El presente trabajo propone la implementación de un plan de manejo de residuos sólidos peligrosos y no peligrosos en la empresa ABRASIVOS S.A. (ASA) para una eficiente gestión de sus insumos y residuos sólidos y por ende el cumplimiento de la normatividad peruana vigente del sector. Se realizaron entrevistas y encuestas al personal a fin de tener un punto de partida del conocimiento y predisposición en la implementación del Plan. Se analizaron los procesos productivos de la empresa así como el diagnóstico técnico operativo del sistema de manejo de residuos sólidos, la identificación de los aspectos y valoración de los impactos ambientales de los procesos de producción a fin de identificar los impactos ambientales significativos y su relación con la generación de residuos sólidos. Esta etapa fue enmarcada en la norma internacional ISO 14001:2004 y una futura implementación de la citada norma en la Empresa. También Se proponen indicadores para medir el desempeño operacional y financiero. Así mismo, este plan sirvió para identificar las opciones de minimización de residuos sólidos y posibilidades de implementación de tecnologías más limpias.
\end{abstract}

Palabras Claves: Residuos sólidos, Residuos sólidos peligrosos, Residuos industriales, Reciclaje, Medio Ambiente.

\begin{abstract}
The paper propose the implementation of a Solid Waste Management Plan in Abrasivos S.A. (ASA) Company that allows the meeting of the Peruvian legal framework and efficient management of supplies and solid waste. Interviews and surveys staff to have a knowledge base and willingness to implement the Plan. We analyzed the processes within the business and technical - operational diagnosis of the system of solid waste management, aspects identification and environmental impacts assessment of production processes to identify significant environmental impacts and their relationship to the generation of solid waste. This stage was framed by international standard ISO 14001:2004 and the future implementation of this standard in the Company. Proposed indicators to measure operational and financial performance. Also, this plan was used to identify options for minimizing solid waste and possibilities for implementation of cleaner technologies.
\end{abstract}

Key words: Solid waste, hazardous solid wastes, industrial wastes, Recycling, Environment.

\section{Introducción}

El notable desarrollo económico e industrial que viene teniendo el Perú durante los últimos años ha aumentado considerablemente la generación de residuos industriales, afectando no sólo al ambiente, sino también la calidad de vida de la población. En el Perú la gestión y el manejo de los residuos sólidos de origen industrial son regulados, fiscalizados y sancionados por los ministerios u organismos regulatorios o de fiscalización correspondientes. Los residuos industriales son definidos por la Ley de Residuos Sólidos ${ }^{1}$ como aquellos residuos generados en las actividades de las diversas ramas industriales, tales como: manufacturera, minera, química, energética, pesquera y otras similares.

El camino para realizar una gestión adecuada de los residuos sólidos peligrosos y no peligrosos, parte de saber qué tipo de residuos se generan, tanto cualitativa como cuantitativamente. Este análisis permite generar diversas alternativas de solución, las cuales son atractivas tanto con el aspecto económico como en el aspecto ambiental; ya que disminuyen la contaminación de suelos producida por la mala disposición de los residuos sólidos, aumenta la vida media de los rellenos sanitarios $\mathrm{y}$ genera puestos de trabajo. Monge $^{2}$ nos menciona que

\footnotetext{
${ }^{1}$ Ing. Ambiental - Universidad Nacional Agraria La Molina, Lima, Perú.

${ }^{2}$ Ing. Ambienta 1- Universidad Nacional Agraria La Molina, Lima, Perú.

${ }^{3}$ Ing. Agrícola - Dpto. Física - Universidad Nacional Agraria La Molina, Lima, Perú. E-mail: wbq@lamolina.edu.pe

${ }^{4}$ Mg. Quím. - Dpto. Química - UUniversidad Nacional Agraria La Molina, Lima, Perú. E-mail: mcesare@lamolina.edu.pe
} 
"Es importante tener en cuenta que los problemas de los residuos sólidos peligrosos y no peligrosos no pueden resolverse simplemente enterrándolos en el suelo. Se debe poner énfasis fundamentalmente en la minimización de residuos, con lo cual se reduce el volumen y la carga contaminante de los desechos, se generan subproductos que se venden como materia prima y se ahorra en la compra de insumos y en el pago de los servicios públicos por el manejo de los residuos.

Como base se debe tomar a la normativa vigente que nos habla sobre los Planes de Manejo de Residuos Sólidos, los que son aplicables a nivel nacional y para todos los subsectores de producción (Industria, Pesquería, Minería, Salud, etc. $)^{3}$. Así también, se aplican a todas las actividades, procesos y operaciones que tengan relación directa con la generación de residuos sólidos, desde que se generan hasta su disposición final técnico - ambiental, incluyendo las distintas fuentes de generación ${ }^{4}$.

El trabajo se desarrolló en las instalaciones de Abrasivos S.A. $(\mathrm{ASA})^{1}$, empresa industrial, líder en la fabricación de abrasivos flexibles en el Perú, abasteciendo mayoritariamente al mercado nacional y exportando más del 30\% de su producción a Latinoamérica, Estados Unidos y Canadá.

En el mes de marzo del 2009 ASA recibe una comunicación de la EPS-RS Navarro Delgado S.R.L, transmitiendo una notificación del Relleno Sanitario Huaycoloro, administrado por PETRAMAS SAC, donde mencionan que ASA ha venido disponiendo residuos que son peligrosos (resinas líquidas), siendo Huaycoloro un relleno no autorizado para tratar tales residuos.

Es por ello que se propone el plan de manejo de residuos sólidos peligrosos y no peligrosos para la empresa, como una herramienta para el manejo adecuado de los residuos sólidos.

\section{Parte experimental}

El estudio se desarrolló en las instalaciones de la empresa Abrasivos S.A. ubicada en la Av. Tomas Alva Edison $\mathrm{N}^{\mathrm{o}}$ 168, en el distrito de Ate, provincia y departamento de Lima.

\section{Análisis de los procesos de manufactura de abrasivos \\ En la actualidad se considera prácticamente imposible cuantificar los materiales químicos empleados como materia prima en los diferentes procesos industriales que se dan en un pais ${ }^{5}$. En el presente trabajo se utilizó como herramienta el mapeo de procesos; según la cual, en cada etapa de los procesos se analizan las entradas, salidas y los residuos generados por las actividades de la empresa ASA.}

Identificación de aspectos y evaluación de impactos ambientales Sobre el contexto de un Sistema de Gestión Ambiental basado en la norma internacional ISO
14001:20046 , se realizó la Identificación de Aspectos e impactos Ambientales de los procesos de ASA con énfasis en la generación de residuos sólidos y los posibles impactos que puedan acarrear. Para ello se elaboró el mapa de procesos y se identificaron los aspectos ambientales del tipo real y potencial. La identificación de los aspectos e impactos relacionados se realizó entendiéndose que entre ambos existe una relación de causa - efecto.

La evaluación de la significancia de cada aspecto e impacto, se determinó usando la siguiente ecuación:

$$
\mathbf{E S}=(\mathbf{C L}+\mathbf{F O}+\mathrm{SI}+\mathbf{I I}+\mathbf{G C})
$$

\section{Donde:}

CL Cumplimiento Legal; FO Frecuencia de Ocurrencia; SI Severidad del Impacto; II Intensidad del Impacto; GC Grado de Control.

La valoración de cada criterio de significancia se realizó mediante tres niveles: bajo, medio y alto, cuyos valores asignados son de 1, 2 y 3 respectivamente.

Le consideró como un aspecto ambiental significativo aquel que tenga un valor igual o mayor a 9 ó donde el umplimiento legal sea igual a un valor de 3 .

\section{Determinación de la peligrosidad de residuos sólidos por línea de producción}

Los procesos industriales generan una gama de residuos peligrosos de diferente naturaleza que tienen características corrosivas, reactivas, explosivas y tóxicas que presentan riesgos potenciales a la salud humana y al ambiente ${ }^{7}$. La identificación y determinación de la peligrosidad de los residuos generado por ASA se desarrolló mediante los siguientes pasos:

Revisión de Hojas de Seguridad: La información generada en el Mapeo de Procesos fue cruzada con la información de las hojas de seguridad a fin de conocer las características del producto.

Valoración de la Peligrosidad: La información del producto fue contrastada con las características que confieren la peligrosidad establecidas en el Anexo 6 del Reglamento de la Ley General de Residuos Sólidos ${ }^{8}$

\section{Clasificación y caracterización de los residuos sólidos}

La clasificación de los residuos sólidos se hizo por su estado físico. Esta metodología ha sido probada en diferentes investigaciones con aplicación a industrias, municipalidades a nivel nacional, empresas privadas y por ONG's ampliamente reconocidas. Posteriormente se clasificaron los residuos generados por ASA en base a su peligrosidad, y por ende se obtuvieron sólo dos tipos de residuos: Peligrosos y No Peligrosos ${ }^{9}$. Para ello se recolectaron los residuos por áreas de producción y se cuantifico la producción total de la empresa. 
El estudio de caracterización se llevó a cabo en la mañana del día siguiente al día generado, tomándose 8 días consecutivos, descartándose la muestra del primer día, debido a que se desconoce la cantidad de residuos que se han almacenado en días anteriore ${ }^{10}$. Cabe señalar que los residuos clasificados como Peligrosos solamente fueron pesados y no fueron caracterizados.

La composición de los residuos sólidos se realizó por clasificación manual. Se llevó a cabo el pesaje y registro de los datos diariamente obteniéndose la composición física en porcentaje de peso por cada residuo, para lo cual se aplicó la siguiente ecuación:

$$
\text { Componente }=\frac{\text { Peso componente separado }}{\text { Peso total de los residuos solidos }} * 100
$$

Se plantearon indicadores de Gestión, con los cuales se puede conocer la relación entre la cantidad de residuos generados respecto a la cantidad de lija producida, dato ayudará a presupuestar costos de tratamiento y/o disposición final. Cabe señalar que los indicadores se utilizan para la toma de decisiones y el mejoramiento continuo, por ello, representan una herramienta gerencial ${ }^{11}$. Se obtienen a través del análisis de actividades y resultan de relacionar cantidades prefijadas para obtener valores determinados.

\section{Identificación de opciones de minimización de residuos sólidos y posibilidades de implementación de tecnologías limpias}

La aplicación de buenas prácticas de gestión de operaciones se basa en la aplicación de una serie de procedimientos y/o políticas organizacionales y administrativas destinadas a mejorar y optimizar los procesos productivos y a promover la participación del personal en actividades destinadas a lograr la minimización de los residuos sólidos ${ }^{12}$, por lo que se proponen opciones de minimización de residuos sólidos, aplicando las $3 R^{\prime} s$ (reducir, reciclar y reutilizar), los que permitirán aprovechar residuos que antes eran desechados.

\section{Resultados y discusión}

I. Análisis de los procesos de manufactura de abrasivos

Abrasivos S.A. divide sus operaciones en 4 grandes áreas: producción, almacenes, laboratorio y el área de Administración, los cuales se aprecian en la Figura 1. A continuación se describe los procesos producidos ${ }^{13}$

\section{Producción}

Incluye las principales líneas de producción de lijas de la Empresa.

Molienda: Destinada a la limpieza, selección, clasificación y mezclado de minerales abrasivos (Oxido de Aluminio, Carburo de Silicio, Granate, Cuarzo, etc.)

Mezclas: Formula adhesivos a base de polímeros para las líneas de Apresto, Discos, Laminaciones y Flexibles.
Apresto: Aplica adhesivos sobre tejidos de algodón quedando impermeabilizados y listos para ser utilizados en Flexibles.

Flexibles: Línea principal de la empresa dedicada a la fabricación de Jumbos de Lija de ancho variable (desde 24 hasta 37 pulgadas de ancho) los cuales son curados en Hornos

Discos: Se producen lijas en forma de discos, utilizando insumos específicos como resina fenólica, alcohol industrial, pigmentos.

Conversión: Destinada a la transformación de los jumbos de lija en producto terminado.

Laminaciones: Proceso de laminado de rollos de abrasivo en los cuales se les aplica el sistema de PSA (Pressure Sensitive Adhesive) o Velcro (Velour / Croche).

\section{Almacenes}

\section{Almacén de Materias Primas (MP)}

Almacena materia prima e insumos. Los residuos de parihuelas son reutilizados para empacar los productos terminados en el almacén de productos terminados o Adex.

\section{Almacén de Productos Terminados (PT)}

Dedicada al almacenaje de los productos terminados listos para la venta. Se subdivide en: Almacén de Productos Terminados Locales y Almacén de Productos Terminados Exportaciones (ADEX).

\section{Laboratorio}

\section{Laboratorio de Control de Calidad}

Durante el ensayo de mineral para grano fino por sedimentación se obtiene como residuos: mineral sedimentado; residuos de metanol, alcohol y agua destilada, que son reaprovechados para la limpieza del laboratorio. Durante el ensayo de mineral para grano grueso según Norma FEPA y según Norma ANSI se producen como residuos el polvo del mineral y el mineral húmedo, ambos residuos peligrosos.

\section{Laboratorio de Ensayos}

En esta etapa se obtiene como residuo el polvo de mineral de acero y aluminio.

\section{Identificación de aspectos y evaluación de impactos ambientales}

La evaluación se efectuó mediante el análisis aspecto criterio, la interrelación de cada aspecto con cada uno de los criterios elegidos, obteniéndose valores cuantitativos como alto, medio o bajo. Seguidamente se ponderó estos valores para determinar la significancia o no de los aspectos ambientales. En esta etapa se reconoció a la generación de residuos sólidos peligrosos y no peligrosos como un Aspecto ambiental recurrente en todas las etapas de producción evaluadas. Ver cuadro 1 


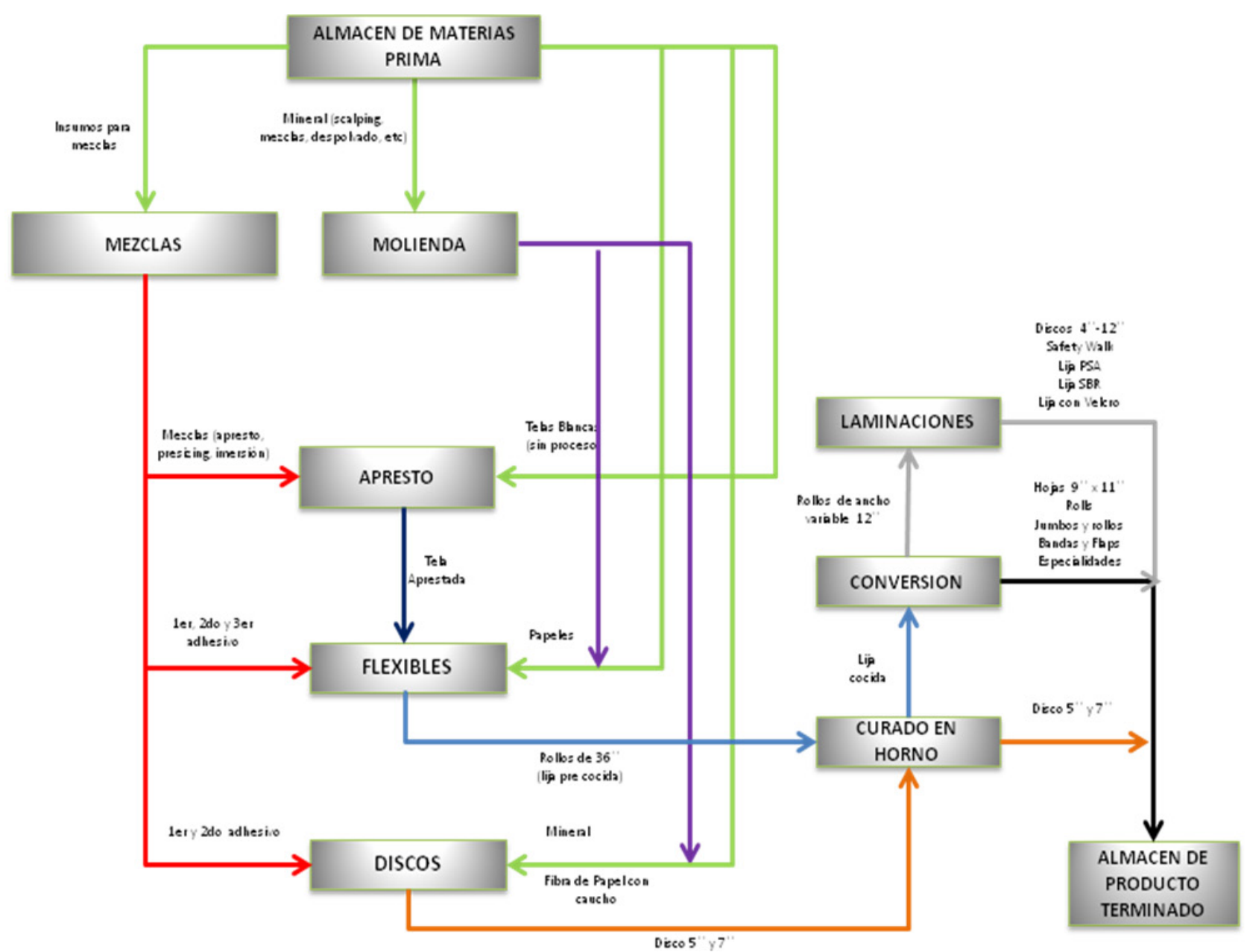

Figura 1. Flujograma integrado del proceso de producción de ASA.

Fuente: Elaboración propia

\section{II.Determinación de la peligrosidad de residuos sólidos por línea de producción}

De la evaluación se rescata que los residuos líquidos peligrosos son: Resina fenólica, resina alquídica, resina acrílica vinílica, resina epóxica, resina acrílica estirenada, Caucho SBR, Alcohol isopropílico, Alcohol industrial. Ver cuadro 2.

III. Diagnóstico del actual sistema de manejo de residuos, complementado con la clasificación y caracterización de residuos sólidos

El principal problema para la empresa radica en que los residuos sólidos no son segregados en la fuente de generación, de modo que no se conoce al detalle qué residuos se generan en cada etapa de sus procesos, ni se distinguen residuos peligrosos de los no peligrosos. A fin de determinar cuantitativamente la generación de residuos sólidos por áreas, se procedió a la clasificación y caracterización de los mismos para evaluar cantidades, volúmenes y peligrosidad de acuerdo a cada área.

\section{Clasificación y Caracterización de los Residuos Sólidos.}

Se basó en la clasificación de los residuos por estado físico. La recolección de muestras se realizó durante 8 días consecutivos. El pesaje fue realizado el mismo día de su generación. Los datos promedios que se obtuvieron por cada área fueron registrados en $\mathrm{kg} /$ día.

Almacenes: E1 93.86 \% de los residuos sólidos generados son cilindros de características peligrosas, producto de la actividad de trasvase de cilindros a contenedores de menor tamaño para la utilización de resinas y solventes. 
Cuadro 1. Identificación y evaluación de aspectos ambientales significativos.

\begin{tabular}{|c|c|c|}
\hline $\begin{array}{c}\text { Aspecto } \\
\text { ambiental } \\
\text { significado }\end{array}$ & $\begin{array}{l}\text { Ampacto } \\
\text { ambiental } \\
\text { significado }\end{array}$ & $\begin{array}{c}\text { ACTIVIDAD / } \\
\text { ETAPA }\end{array}$ \\
\hline \multirow{8}{*}{$\begin{array}{l}\text { Consumo } \\
\text { de energía } \\
\text { eléctrica }\end{array}$} & \multirow{8}{*}{$\begin{array}{l}\text { Disminución } \\
\text { de la energía } \\
\text { eléctrica para } \\
\text { otros usos }\end{array}$} & ALMACENES \\
\hline & & MEZCLAS \\
\hline & & APRESTO \\
\hline & & MOLIENDA \\
\hline & & DISCOS \\
\hline & & CONVERSIÓN \\
\hline & & LAMINADO \\
\hline & & FLEXIBLES \\
\hline \multirow[t]{2}{*}{$\begin{array}{l}\text { Consumo de } \\
\text { GN }\end{array}$} & \multirow{2}{*}{$\begin{array}{l}\text { Agotamiento } \\
\text { de recursos } \\
\text { naturales }\end{array}$} & FLEXIBLES \\
\hline & & ALMACENES \\
\hline \multirow{4}{*}{$\begin{array}{c}\text { Emanación de } \\
\text { COV's }\end{array}$} & \multirow{4}{*}{$\begin{array}{c}\text { Contaminación } \\
\text { del aire }\end{array}$} & MEZCLAS \\
\hline & & APRESTO \\
\hline & & LAMINADO \\
\hline & & FLEXIBLES \\
\hline \multirow{5}{*}{$\begin{array}{c}\text { Emisión } \\
\text { de material } \\
\text { particulado }\end{array}$} & \multirow{5}{*}{$\begin{array}{c}\text { Contaminación } \\
\text { del aire }\end{array}$} & ALMACENES \\
\hline & & MEZCLAS \\
\hline & & MOLIENDA \\
\hline & & DISCOS \\
\hline & & FLEXIBLES \\
\hline \multirow{8}{*}{$\begin{array}{l}\text { Generación } \\
\text { de Residuos } \\
\text { Sólidos No } \\
\text { Peligrosos }\end{array}$} & \multirow{8}{*}{$\begin{array}{l}\text { Reducción de } \\
\text { la vida útil } \\
\text { del relleno } \\
\text { sanitario }\end{array}$} & ALMACENES \\
\hline & & MEZCLAS \\
\hline & & APRESTO \\
\hline & & MOLIENDA \\
\hline & & DISCOS \\
\hline & & CONVERSIÓN \\
\hline & & LAMINADO \\
\hline & & FLEXIBLES \\
\hline \multirow{6}{*}{$\begin{array}{l}\text { Generación } \\
\text { de Residuos } \\
\text { Sólidos } \\
\text { Peligrosos }\end{array}$} & \multirow{6}{*}{$\begin{array}{l}\text { Reducción de } \\
\text { la vida útil } \\
\text { del relleno de } \\
\text { seguridad }\end{array}$} & ALMACENES \\
\hline & & MEZCLAS \\
\hline & & APRESTO \\
\hline & & DISCOS \\
\hline & & LAMINADO \\
\hline & & FLEXIBLES \\
\hline \multirow{3}{*}{$\begin{array}{c}\text { Potencial } \\
\text { derrame de } \\
\text { elementos } \\
\text { contaminantes }\end{array}$} & \multirow{2}{*}{$\begin{array}{l}\text { Contaminación } \\
\text { del suelo }\end{array}$} & ALMACENES \\
\hline & & MEZCLAS \\
\hline & \multirow{8}{*}{$\begin{array}{c}\text { Agotamiento } \\
\text { de los recursos } \\
\text { naturales }\end{array}$} & ALMACENES \\
\hline \multirow{7}{*}{$\begin{array}{l}\text { Potencial } \\
\text { incendio }\end{array}$} & & MEZCLAS \\
\hline & & APRESTO \\
\hline & & MOLIENDA \\
\hline & & DISCOS \\
\hline & & CONVERSIÓN \\
\hline & & LAMINADO \\
\hline & & FLEXIBLES \\
\hline
\end{tabular}

Fuente: elaboración propia
Apresto: Se genera $48.23 \mathrm{~kg}$ /día de Trimas de tela, siendo el $81.33 \%$, producto de la actividad de embobinado; seguido de tucos de cartón $3.71 \mathrm{~kg} /$ día.

Así mismo, como parte de la limpieza diaria de las máquinas, se generan residuos líquidos de solventes, alcohol industrial con agua y residuos de resinas. La generación aproximada es de 01 cilindro semanal de aproximadamente $150 \mathrm{~kg}$.

Conversión: Se generan casi un $60.00 \%$ de trimas de lijas, seguido por un $18.38 \%$ de lijas. Respecto a plásticos un $7.01 \%$. Ya en menor cantidad cola, cerca de $3.00 \%$.

Discos: El principal residuo generado es resina fenólica, representando un $43.50 \%$ con una tasa de $4.29 \mathrm{~kg} /$ día, las mismas que son dispuestas en bolsas dentro de cilindros.

Flexibles: Los principales residuos generados son resinas, tanto fenólica como resina de úrea. En el caso de la primera se genera como resina líquida que se mezcla con resina epóxica y se estabiliza, solidificándola; la tasa de generación diaria es de $79 \mathrm{~kg} /$ día, siendo el $45.47 \%$ de los residuos generados en el área. La resina de úrea es generada $38.43 \mathrm{~kg} /$ día, representando el $21.92 \%$. Otro residuo a considerar son las lijas defectuosas, como parte del control de calidad que representan el $20.88 \%$ $(36.61 \mathrm{~kg} / \mathrm{día})$ del total.

Laboratorios: Los residuos generados en el laboratorio Técnico son trimas de lijas en una tasa de $2.81 \mathrm{~kg} / \mathrm{día}$ siendo un $20.41 \%$ del total generado. Mientras que en el Laboratorio de Control de Calidad, los principales residuos son telas, que representan un $35.38 \%(4.88 \mathrm{~kg} /$ día), seguido son los plásticos (envases utilizados para los ensayos) y papel látex que suman aproximadamente un $32.50 \%(2.06 \mathrm{~kg} /$ día y $2.40 \mathrm{~kg} /$ día respectivamente). Todos los residuos están contaminados con diferentes insumos como Estearato de Zinc (polvo), Resinas epóxicas y fenólicas, Pigmentos, Cola animal, mezclas de adhesivo seco entre otros insumos. También se generan en menos cantidad lijas (1.64 kg/día).

Mantenimiento: Residuos de chatarra, siendo el mayor porcentaje de todo lo generado $8.04 \mathrm{~kg} /$ día cerca del $87 \%$.

Mezclas: Tiene como principal residuo los plásticos, 6.29 $\mathrm{kg} / \mathrm{día}$, representando un $47.16 \%$. Un residuo importante a considerar son los papeles y plásticos contaminados con mezclas de resinas, las cuales llegan al 23.85\%.

Molienda: Residuos de mineral, 13.44 kg/día (70.28\%). Los minerales usados en la semana de caracterización fueron Carburo de Silicio y Oxido de Aluminio. 
Cuadro 2. Evaluación de Peligrosidad de Residuos.

\begin{tabular}{|c|c|c|c|c|c|c|c|c|c|c|}
\hline Insumo & Corrosivo & Reactivo & Explosivo & Tóxico & Inflamable & Patógeno & $\begin{array}{l}\text { Condiciones de } \\
\text { Almacenamiento }\end{array}$ & Estado & $\begin{array}{l}\text { Medidas de Lucha } \\
\text { contra incendio }\end{array}$ & Incompatibilidad \\
\hline Resina Fenólica & $\mathbf{S i}$ & No & No & Si & No & No & $\begin{array}{c}\text { Lugar fresco y seco, protegido } \\
\text { de los rayos solares }\end{array}$ & Liquido & $\begin{array}{c}\text { Refrigerar los } \\
\text { contenedores } \\
\text { expuestos al fuego }\end{array}$ & No ácidos \\
\hline $\begin{array}{l}\text { Resina Urea } \\
\text { formaldehido }\end{array}$ & $\mathrm{N} / \mathrm{E}$ & $\mathrm{N} / \mathrm{E}$ & $\mathrm{N} / \mathrm{E}$ & $\mathrm{N} / \mathrm{E}$ & $\mathrm{N} / \mathrm{E}$ & $\mathrm{N} / \mathrm{E}$ & $\begin{array}{l}\text { Lugares ventilados bajo } \\
\text { sombra, } \mathrm{T}<25^{\circ} \mathrm{C}\end{array}$ & $\begin{array}{l}\text { Solución } \\
\text { acuosa }\end{array}$ & $\begin{array}{l}\text { Agua, espuma y } \\
\text { CO2 }\end{array}$ & $\mathrm{N} / \mathrm{E}$ \\
\hline Resina Alquidica & No & No & No & No & $\mathbf{S i}$ & No & $\mathrm{N} / \mathrm{E}$ & $\mathrm{N} / \mathrm{E}$ & $\mathrm{N} / \mathrm{E}$ & $\mathrm{N} / \mathrm{E}$ \\
\hline $\begin{array}{l}\text { Resina acrílica } \\
\text { vinílica }\end{array}$ & No & No & No & No & $\mathbf{S i}$ & No & $\mathrm{N} / \mathrm{E}$ & $\mathrm{N} / \mathrm{E}$ & $\mathrm{N} / \mathrm{E}$ & $\mathrm{N} / \mathrm{E}$ \\
\hline Resina Epóxica & No & No & No & Si & No & No & $\begin{array}{l}\text { Mantenga los contenedores } \\
\text { bien cerrados cuando no se } \\
\text { están usando }\end{array}$ & $\begin{array}{l}\text { Líquido } \\
\text { semisólido }\end{array}$ & $\mathrm{CO} 2$ y PQS & $\begin{array}{l}\text { Ácidos. Aminas. } \\
\text { Bases. Agentes } \\
\text { oxidantes }\end{array}$ \\
\hline $\begin{array}{l}\text { Resina acrílica } \\
\text { estirenada }\end{array}$ & No & No & No & No & Si & No & $\begin{array}{l}\text { Lugares frescos, no dejar } \\
\text { recipientes abiertos }\end{array}$ & $\begin{array}{l}\text { Emulsión } \\
\text { viscosa } \\
\text { blanquecina }\end{array}$ & $\begin{array}{l}\text { Agua, espuma y } \\
\text { CO2 }\end{array}$ & $\mathrm{N} / \mathrm{E}$ \\
\hline Estearato de Zinc & $\mathrm{N} / \mathrm{E}$ & $\mathrm{N} / \mathrm{E}$ & $\mathrm{N} / \mathrm{E}$ & $\mathrm{N} / \mathrm{E}$ & $\mathrm{N} / \mathrm{E}$ & $\mathrm{N} / \mathrm{E}$ & $\begin{array}{c}\text { Lugares ventilados, lejos de } \\
\text { llamas }\end{array}$ & $\begin{array}{l}\text { Polvo de color } \\
\text { blanco }\end{array}$ & $\begin{array}{l}\text { PQS, CO2, Agua a } \\
\text { presión, espuma }\end{array}$ & $\begin{array}{l}\text { Agentes oxidantes } \\
\text { fuertes, peróxido, } \\
\text { ácidos, bases y } \\
\text { oxígeno }\end{array}$ \\
\hline Caucho SBR & No & No & No & No & $\mathbf{S i}$ & No & $\begin{array}{l}\text { Lejos del calor, fuentes de } \\
\text { ignición y llamas }\end{array}$ & Líquido & $\begin{array}{l}\text { Agua, espuma y } \\
\text { CO2 }\end{array}$ & $\mathrm{N} / \mathrm{E}$ \\
\hline $\begin{array}{l}\text { Etanol - OH } \\
\text { industrial }\end{array}$ & No & No & $\begin{array}{l}\text { Las mezclas } \\
\text { vapor/ } \\
\text { aire son } \\
\text { explosivas. }\end{array}$ & No & $\mathbf{S i}$ & No & $\begin{array}{l}\text { A prueba de incendio. } \\
\text { Separado de oxidantes } \\
\text { fuertes. Mantener un lugar } \\
\text { bien ventilada. }\end{array}$ & $\begin{array}{l}\text { Líquido } \\
\text { incoloro } \\
\text { de olor } \\
\text { característico }\end{array}$ & $\begin{array}{l}\text { Polvo, Agua, } \\
\text { espuma y CO2 }\end{array}$ & Oxidantes fuertes \\
\hline Alcohol Isopropílico & No & No & $\begin{array}{l}\text { Las mezclas } \\
\text { vapor/ } \\
\text { aire son } \\
\text { explosivas. }\end{array}$ & No & $\mathbf{S i}$ & No & $\begin{array}{l}\text { A prueba de incendio. } \\
\text { Separado de oxidantes } \\
\text { fuertes. Mantener un lugar } \\
\text { bien ventilada }\end{array}$ & $\begin{array}{l}\text { Liquido } \\
\text { incoloro, } \\
\text { claro }\end{array}$ & $\begin{array}{l}\text { Polvos, espuma } \\
\text { resistente al } \\
\text { alcohol, agua } \\
\text { en grandes } \\
\text { cantidades, } \\
\text { dióxido de } \\
\text { carbono. }\end{array}$ & $\mathrm{N} / \mathrm{E}$ \\
\hline $\begin{array}{l}\text { Sulfato de Bario } \\
\text { (Baritina) }\end{array}$ & No & No & No & No & No & No & $\begin{array}{l}\text { Lugar abierto, seco y } \\
\text { ventilado, lejos de sustancias } \\
\text { alimenticias. }\end{array}$ & $\begin{array}{l}\text { Polvo fino } \\
\text { de color } \\
\text { grisáceo }\end{array}$ & $\mathrm{N} / \mathrm{E}$ & $\mathrm{N} / \mathrm{E}$ \\
\hline
\end{tabular}

Fuente: elaboración propia 
Generación de Indicadores de Gestión de Residuos: Con la información obtenida, respecto a los kilogramos de residuos generados en las diferentes áreas durante la semana de caracterización, en la Tabla 1 se muestran los indicadores expresados en $\mathrm{kg}$ residuos generados / metro de lija producida en las cuatro áreas donde se produce mayor cantidad de residuos. Por ejemplo en el área de Conversión se generan Trimas de Lijas, así por cada metro de lija producida se generan 0.01 kilogramos de trimas, lo que equivale a un $1.23 \%$, porcentaje que está dentro del $5.00 \%$ permitido en los procesos de producción (dato referencia en líneas de producción para generación de mermas).

Tabla 1. Indicadores de desempeño ambiental según tipo de residuos de mayor generación por área.

\begin{tabular}{|c|c|c|c|c|c|}
\hline Área & $\begin{array}{c}\text { Lija } \\
\text { producida }^{(a)} \\
(\mathrm{m} / \mathrm{sem})\end{array}$ & Residuo & $\begin{array}{c}\text { Residuos } \\
\text { Generados }^{(b)} \\
(\mathrm{Kg} / \mathrm{sem}) \\
\end{array}$ & $\begin{array}{c}\text { Indicador }^{(a / b)} \\
(\mathrm{Kg} / \mathrm{m})\end{array}$ & $\%$ \\
\hline \multirow[t]{2}{*}{ Flexibles } & & Resina & & & \\
\hline & 69300.00 & $\begin{array}{c}\text { Fenólica } \\
\text { con Epóxica }\end{array}$ & 558.00 & 0.01 & 0.81 \\
\hline Conversión & 94941.00 & $\begin{array}{c}\text { Trima de } \\
\text { Lija }\end{array}$ & 1171.63 & 0.01 & 1.23 \\
\hline Apresto & 74000.00 & $\begin{array}{c}\text { Trima de } \\
\text { Tela }\end{array}$ & 337.60 & 0.00 & 0.46 \\
\hline Discos & 27700.00 & $\begin{array}{l}\text { Resina } \\
\text { fenólica }\end{array}$ & 30.00 & 0.00 & 0.11 \\
\hline
\end{tabular}

Fuente: elaboración propia

\section{Comercialización y Reaprovechamiento}

El mayor ingreso por venta de residuos en ASA es generado por la venta de cilindros, ya que se venden a $\mathrm{S} / .15 .00$ por unidad; por la venta de baldes de pintura se obtienen $\mathrm{S} /$. 1.00 por unidad; y finalmente por la venta de parihuelas de madera se obtiene un precio entre S/. 2.00 y S/. 5.00. El ingreso promedio mensual aproximado es de $\mathrm{S} / .1800 .00$.

\section{Disposición Final de los Residuos}

Los residuos comunes y de oficina son recolectados a través de la Municipalidad de Ate una vez por semana. La recolección de los residuos sólidos, tanto peligrosos como no peligrosos se realiza a través de una EPS-RS y son dispuestos en el Relleno sanitario de Huaycoloro.

El costo total promedio mensual de disposición de residuos es de S/. 2248.88.

\section{Propuesta para el manejo de residuos sòlidos en la} fábrica de Abrasivos S.A.

\section{Producción de residuos sólidos}

En la Tabla 2 se aprecia la composición promedio de residuos generados al día en la planta de Abrasivos S.A, siendo los principales residuos sólidos generados las trimas de lijas (31.31\%), resinas epóxicas-fenólica sólida $(14.66 \%)$, lijas defectuosas $(8.40 \%)$, cilindros metálicos para venta $(6.70 \%)$.
Tabla 2. Producción Total de Residuos Sólidos en ASA.

\begin{tabular}{|c|c|c|c|c|}
\hline $\begin{array}{l}\text { Resumen de } \\
\text { Residuos }\end{array}$ & Tipo & $\begin{array}{c}\text { Total } \\
\mathrm{kg} / \text { día }\end{array}$ & $\begin{array}{c}\text { Total } \\
\text { tn / mes }\end{array}$ & Porcentaje \\
\hline Papel Látex, bolsas & NP & 37,71 & 1,13 & $6,94 \%$ \\
\hline Papel de Oficina & NP & 37,71 & 1,13 & $6,94 \%$ \\
\hline Plástico & NP & 37,71 & 1,13 & $6,94 \%$ \\
\hline Vidrio & NP & 37,71 & 1,13 & $6,94 \%$ \\
\hline Trimas de Lijas & NP & 37,71 & 1,13 & $6,94 \%$ \\
\hline Lijas & NP & 37,71 & 1,13 & $6,94 \%$ \\
\hline Trimas de tela & NP & 37,71 & 1,13 & $6,94 \%$ \\
\hline Cartones & NP & 37,71 & 1,13 & $6,94 \%$ \\
\hline Tucos & NP & 37,71 & 1,13 & $6,94 \%$ \\
\hline Telas / trapos & NP & 37,71 & 1,13 & $6,94 \%$ \\
\hline Tela aprestada & NP & 37,71 & 1,13 & $6,94 \%$ \\
\hline Cilindros & NP & 37,71 & 1,13 & $6,94 \%$ \\
\hline Epp's & NP & 37,71 & 1,13 & $6,94 \%$ \\
\hline Parihuelas & NP & 37,71 & 1,13 & $6,94 \%$ \\
\hline $\begin{array}{l}\text { Resinas fenólica } \\
\text { Líquidas }\end{array}$ & NP & 37,71 & 1,13 & $6,94 \%$ \\
\hline Resinas úrea Sólida & NP & 37,71 & 1,13 & $6,94 \%$ \\
\hline $\begin{array}{l}\text { Resina Fenólica } \\
\text { epóxica Sólida }\end{array}$ & NP & 37,71 & 1,13 & $6,94 \%$ \\
\hline Cola & NP & 37,71 & 1,13 & $6,94 \%$ \\
\hline Aceite & NP & 37,71 & 1,13 & $6,94 \%$ \\
\hline $\begin{array}{l}\text { Plástico } \\
\text { contaminado }\end{array}$ & NP & 37,71 & 1,13 & $6,94 \%$ \\
\hline $\begin{array}{l}\text { Papeles } \\
\text { contaminados }\end{array}$ & NP & 37,71 & 1,13 & $6,94 \%$ \\
\hline $\begin{array}{l}\text { Trapos } \\
\text { contaminados }\end{array}$ & NP & 37,71 & 1,13 & $6,94 \%$ \\
\hline Lijas contaminadas & NP & 37,71 & 1,13 & $6,94 \%$ \\
\hline Orgánicos & NP & 37,71 & 1,13 & $6,94 \%$ \\
\hline Chatarra & NP & 37,71 & 1,13 & $6,94 \%$ \\
\hline Residuos comunes & NP & 37,71 & 1,13 & $6,94 \%$ \\
\hline Mineral con tierra & NP & 37,71 & 1,13 & $6,94 \%$ \\
\hline Total & NP & 37,71 & 1,13 & $6,94 \%$ \\
\hline
\end{tabular}

NP No Peligroso

Fuente: elaboración propia

En la Figura 2 se puede apreciar que del $100.00 \%$ de residuos generados, el $82.36 \%$ corresponde a residuos no peligrosos y el $17.64 \%$ a residuos peligrosos.

\section{Generación de Residuos Sólidos}

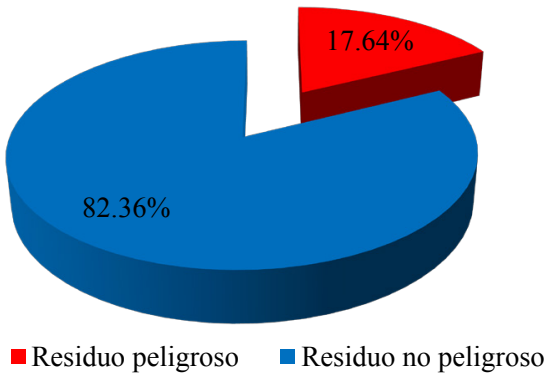

Figura 2. Generación de Residuos Sólidos en ASA. Fuente: elaboración propia 


\section{Manejo de Residuos Sólidos}

Describe las pautas a seguir para realizar un manejo adecuado de los residuos sólidos generados empleando técnicas de minimización, reaprovechamiento, segregación y comercialización; además se definen las consideraciones a tomar para el almacenamiento, transporte y disposición final de los mismos ${ }^{14}$.

\section{Segregación}

Se verifica la correspondencia del tipo de residuos de acuerdo al Código de Colores conforme a la NTP 900.058.2005 2 .

\section{Recolección, Rotulado y Transporte Interno}

La recolección se realiza desde los cilindros de colores destinados para la disposición temporal de los residuos sólidos hacia el almacén de residuos sólidos con una frecuencia de una vez al día al finalizar el turno y siguiendo las rutas de tránsito establecidas por la empresa.

El rotulado de los Residuos Peligrosos se realizará tomando en cuenta que para la mayoría de los residuos de características misceláneas, se deberá considerar para el Rotulado la CLASE 9: Sustancias y objetos peligrosos varios. Respecto al Número de Identificación de la Materia, se considerará la Sustancia con la que está contaminado el residuo / material.

\section{Almacenamiento Central.}

Se propone retirar el contenedor de la EPS-RS debido a que ocupa demasiado espacio y no permite la segregación óptima de los residuos. En su reemplazo, se propone:

- Delimitar un espacio para el almacenamiento de trimas de lijas, debido a que éstas representan el 31.31\% de la producción de residuos en la Empresa.

- Delimitar un espacio para los residuos sólidos peligrosos, los cuales representan el $17.43 \%$ de la generación mensual de los residuos de la Empresa

- Delimitar un espacio para almacenar las resinas de úrea formaldehido estabilizadas con epóxica, debido a que representa el $14.66 \%$.

- Delimitar un espacio para el almacenamiento de los cilindros usados que representan $6.70 \%$ y que son comercializados posteriormente.

El área continua será distribuida en el segundo nivel para las trimas de tela $(8.87 \%)$ y Tucos de cartón $(0.24 \%)$. Mientras que en el primer nivel: Papeles y cartones $(2.12 \%)$ serán almacenados en contenedores especiales debido a que serán donados a una ONG; Plásticos (2.93\%) donde la gran mayoría tipo strecht film; Residuos comunes (0.34) almacenados en un contenedor de plástico con tapa; Chatarra (1.77\%) contenidos dentro de un cilindro para posterior comercialización.

\section{Comercialización o Reaprovechamiento.}

Se propone añadir a la actual comercialización de parihuelas y cilindros, otros residuos mostrados en la Tabla 3. Con esta nueva gestión se disminuirá la cantidad de residuos que ingresan al relleno sanitario y que no son aprovechados, mejorará la imagen de la empresa como una empresa ambientalmente responsable y sensibilizará a la Empresa en el respeto al ambiente.

\section{Disposición Final}

Para los residuos sólidos peligrosos, éstos son llevados al relleno de seguridad autorizado BEFESA y los residuos no peligrosos al relleno sanitario.

En comparación a la gestión actual, se propone cambiar de EPS-RS y de Relleno Sanitario de Disposición Final. En primer lugar, los costos por transporte disminuyen en casi $30.00 \%$ por viaje de S/.650.00 a S/450.00. En segundo lugar, el Relleno Sanitario de RELIMA no permite el ingreso de recicladores, lo que aseguraría que principalmente las trimas de lijas, residuo de mayor importancia para la empresa, no sea reutilizado y vendido en el mercado informal, perjudicando la imagen de la marca. La comparación entre los costos actuales y los costos propuestos se muestran en la Figura 3.

Tabla 3. Ingreso por venta de residuos.

\begin{tabular}{lccc}
\hline Residuo & Ton/mes & $\begin{array}{c}\text { Precio Venta } \\
\text { por Kg }\end{array}$ & $\begin{array}{c}\text { Ingreso } \\
\text { mensual }\end{array}$ \\
\hline $\begin{array}{l}\text { Papel Látex, } \\
\text { bolsas }\end{array}$ & 1.13 & 0.2 & $\mathrm{~S} / .226 .26$ \\
Plástico & 0.48 & 0.2 & $\mathrm{~S} / .95 .64$ \\
Cartones y tucos & 0.15 & 0.2 & $\mathrm{~S} / .30 .00$ \\
Trimas de tela & 1.45 & 5 & $\mathrm{~S} / .260 .00$ \\
Cilindros $(*)$ & 115 & 15 & $\mathrm{~S} / .1,725.00$ \\
Parihuelas & $15^{*}$ & 3.5 & $\mathrm{~S} / .52 .50$ \\
Chatarra & 0.29 & 0.5 & $\mathrm{~S} / .144 .45$ \\
\hline Total & & & $\mathrm{S} / . \mathbf{2 2 5 3 3 . 8 5}$ \\
\hline
\end{tabular}

(*) Se tomó en cuenta la data del año 2009

Fuente: elaboración propia

En la Tabla 4 se aprecia el Plan de Residuos Sólidos propuesto, en el cual se plantean opciones de reciclaje a través de convenios con FUNDADES para los papeles; y con HP, a través de su programa Planet Partners, para los tóner de su marca. 


\section{Costo de disposicion de residuos}

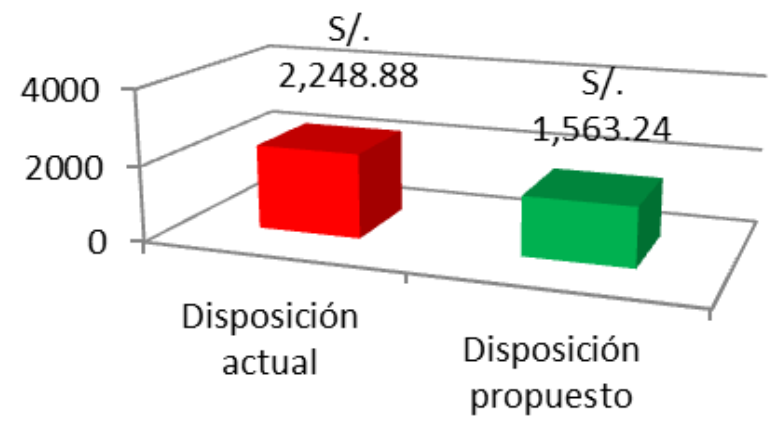

\section{Ingreso por comercializacion de residuos}

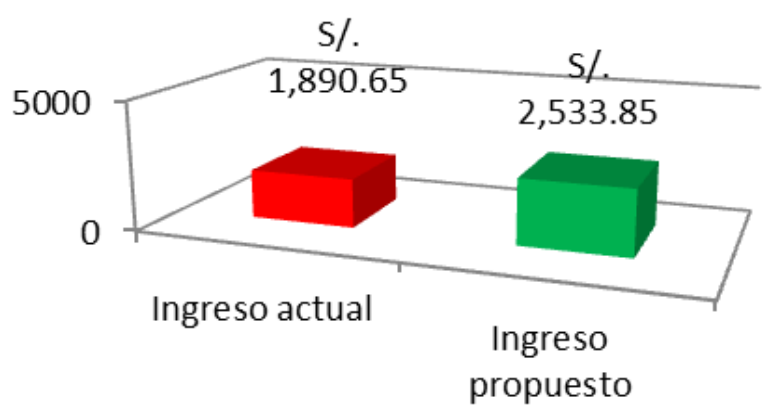

Figura 3. Disposición y comercialización de residuos.

Fuente: elaboración propia

Tabla 4. Plan de Residuos Sólidos propuesto para la empresa Abrasivos S.A.

\begin{tabular}{|c|c|c|c|c|c|}
\hline Residuo & Tipo & Origen & Manejo & Destino & Frecuencia \\
\hline Lijas & NP & Conversión & EPS-RS & Relleno Sanitario & Semanal \\
\hline Resinas sólidas & NP & Flexibles, Apresto & EPS-RS & Relleno Sanitario & Semanal \\
\hline Cilindros & NP & Almacén, Mezclas & EC-RS & Venta & Mensual \\
\hline Parihuelas & NP & Almacén & EC-RS & Venta & Mensual \\
\hline Comunes & NP & SSHH y Comedor & Municipalidad & Relleno Sanitario & Semanal \\
\hline Papeles y Cartones & NP & $\begin{array}{c}\text { Oficinas } \\
\text { administrativas }\end{array}$ & $\begin{array}{c}\text { Reciclaje } \\
\text { FUNDADES }\end{array}$ & Donación & Mensual \\
\hline Tóners & NP & $\begin{array}{c}\text { Oficinas } \\
\text { administrativas }\end{array}$ & Reciclaje HP & Donación & Mensual \\
\hline Resinas Líquidas & $\mathrm{P}$ & $\begin{array}{l}\text { Flexibles, Apresto, } \\
\text { Impresiones }\end{array}$ & EPS-RS & Relleno de Seguridad & Según generación \\
\hline
\end{tabular}

\section{Producción Más Limpia}

En las siguientes tablas se proponen las siguientes actividades para una producción mas limpia.

a) Reusar internamente los desechos

\begin{tabular}{|c|c|c|}
\hline Área & $\begin{array}{l}\text { Residuo } \\
\text { Generado }\end{array}$ & Propuesta \\
\hline Flexibles & $\begin{array}{l}\text { Resina } \\
\text { Formaldehido } \\
\text { (residuo } \\
\text { líquido) }\end{array}$ & $\begin{array}{l}\text { La resina formaldehido } \\
\text { actualmente se reutiliza en la } \\
\text { 1ra máquina de flexibles, ya } \\
\text { que el residuo sale limpio. } \\
\text { Mientras que en la 2da } \\
\text { máquina, no se reutiliza ya } \\
\text { que sale contaminada con } \\
\text { otros componentes. Para } \\
\text { poder reutilizarla, es necesaria } \\
\text { la instalación de un filtro en la } \\
\text { 2da máquina. }\end{array}$ \\
\hline $\begin{array}{l}\text { Discos, } \\
\text { apresto }\end{array}$ & $\begin{array}{l}\text { Alcohol } \\
\text { Industrial } \\
\text { (residuos } \\
\text { líquido) }\end{array}$ & $\begin{array}{l}\text { Previa destilación del solvente } \\
\text { sea nuevamente utilizada para } \\
\text { la limpieza de maquinarias } \\
\text { dentro de planta. }\end{array}$ \\
\hline
\end{tabular}

Fuente: elaboración propia b) Mejorar la gestión y las prácticas de operación

\begin{tabular}{|c|c|c|}
\hline Área & $\begin{array}{l}\text { Residuo } \\
\text { Generado }\end{array}$ & Propuesta \\
\hline $\begin{array}{r}\text { Oficin } \\
\text { Administr }\end{array}$ & Papeles & $\begin{array}{l}\text { Consumir papel para lo } \\
\text { estrictamente necesario, } \\
\text { imprimiendo solo los } \\
\text { documentos que tengan que } \\
\text { estar impresos e utilizar la } \\
\text { impresión por ambas caras. }\end{array}$ \\
\hline $\begin{array}{l}\text { Almacén } \\
\text { de Materias } \\
\text { Primas }\end{array}$ & $\begin{array}{l}\text { Guantes } \\
\text { contaminado } \\
\text { con solvente }\end{array}$ & $\begin{array}{l}\text { Sustituir los guantes } \\
\text { descartables actualmente } \\
\text { usados para el despacho } \\
\text { de Dowanol (solvente) } \\
\text { por guantes perennes de } \\
\text { nitrilo, a fin de reducir la } \\
\text { generación estos residuos. }\end{array}$ \\
\hline
\end{tabular}

Fuente: elaboración propia

c) Mejora del diseño de producto

\begin{tabular}{cll}
\hline Área & \multicolumn{1}{c}{$\begin{array}{c}\text { Residuo } \\
\text { Generado }\end{array}$} & \multicolumn{1}{c}{ Propuesta } \\
\hline & $\begin{array}{l}\text { Trima de } \\
\text { lijas }\end{array}$ & $\begin{array}{l}\text { Las trimas de lijas se } \\
\text { obtienen al cortar las lijas } \\
\text { longitudinalmente por } \\
\text { ambos extremos. Impulsar } \\
\text { el proyecto de introducción } \\
\text { Conversión }\end{array}$ \\
$\begin{array}{c}139.36 \mathrm{~kg} / \\
\text { día. }(4.18\end{array}$ & $\begin{array}{l}\text { en el mercado de lijas con } \\
\text { trimas., a fin de prescindir } \\
\text { del corte de éstas. }\end{array}$ \\
\hline
\end{tabular}


d) Mejorar la gestión y las prácticas de operación

\begin{tabular}{|c|c|c|}
\hline Área & $\begin{array}{c}\text { Residuo } \\
\text { Generado }\end{array}$ & Propuesta \\
\hline Mezclas & Cola & $\begin{array}{l}\text { La cola animal, se } \\
\text { degrada en un máximo de } \\
6 \text { días. Pasado este tiempo } \\
\text { el residuo ya no es útil y } \\
\text { se tiene que disponer. } \\
\text { Se requiere preparar } \\
\text { la mezcla de cola en la } \\
\text { cantidad necesaria para } \\
\text { evitar mermas. }\end{array}$ \\
\hline Flexibles & $\begin{array}{l}\text { Resina } \\
\text { Fenólica }\end{array}$ & $\begin{array}{l}\text { La resina fenólica } \\
\text { combinada con la resina } \\
\text { epóxica forma una masa } \\
\text { sólida. Se recomienda } \\
\text { continuar con esta } \\
\text { práctica. }\end{array}$ \\
\hline
\end{tabular}

Fuente: elaboración propia

\section{Conclusiones}

La Empresa Abrasivos S.A. no cuenta con un Sistema de Gestión Ambiental implementado ni con un Plan de Manejo de residuos sólidos, así como tampoco cumple la Norma Técnica de INDECOPI NTP 900.058 - GESTIÓN AMBIENTAL. Gestión de Residuos. Códigos de colores para los dispositivos de almacenamiento de residuos. En consecuencia no tiene un adecuado manejo y gestión de los residuos sólidos generados en sus procesos de producción.

Del estudio de caracterización realizado se obtuvo que el $82.36 \%$ de los residuos generados son residuos no peligrosos y el $17.64 \%$ son residuos peligrosos. Además, el residuo que se genera en mayor porcentaje son las trimas de lijas (31.31\%), generadas en el área de Conversión, que representan una cantidad mensual de 5.11 ton/mes. Así mismo, en la misma línea, las lijas representan el 8.40\%, que representan una cantidad mensual de 1.37 ton $/$ mes.

Se identificó como una oportunidad de minimización de residuos sólidos la venta de cilindros obtenidos en el área de Almacenes (93.86\% de los residuos generados).

Se identificaron como oportunidades de implementación de Producción Más Limpia: las mejoras en el diseño del producto concernientes a la reducción de la trima de lijas (4.18 ton/mes); mejorar la gestión y las prácticas de operación minimizando optimizando el uso de cola y estabilizando la resina fenólica con la resina epóxica; y en el reuso interno de lo que hoy se considera desechos, tales como resina formaldehido y el alcohol industrial.

Los indicadores de desempeño ambiental referente a la Gestión de Residuos sólidos se encuentran dentro de los valores promedio usados en los procesos de producción (generación de mermas menor a 5\%). Si bien puede ser no muy significativo para Abrasivos S.A en términos de producción, si es considerable en términos de costos por disposición final de residuos.

\section{Agradecimiento}

Nuestro profundo agradecimiento a la empresa Abrasivos S.A. por todo el apoyo brindado en la elaboración y desarrollo de la presente propuesta.

\section{Literatura citada}

Abrasivos, S. A. 2009. Información General. Consultado 06 de setiembre del 2009. Disponible en http://www. asaabrasives.com/index es.htm.

Benavides, L. 1993. Guía para la Definición y Clasificación de Residuos Peligrosos. CEPIS. Lima. Perú.

CEPIS (Centro Panamericano de Ingeniería Sanitaria). 1993. Guía para la definición y clasificación de residuos peligrosos (RESPEL).

Decreto Supremo No 057-2004. 2004. Reglamento Ley $\mathrm{N}^{\circ}$ 27314, Ley General de Residuos Sólidos.

ISO 14001. 2004. Norma Internacional - Sistema de Gestión Ambiental.

Lanegra, I. 2004. Legislación Ambiental en el manejo de los Residuos Sólidos. Desafíos y Herramientas para la Gestión Integral de Residuos. Ciudad Saludable e Instituto para la Calidad. Primera edición. Perú.

Ley $N^{\circ}$ 27314. 2000. Ley General de Residuos Sólidos.

Monge, G. 1998 Seminario internacional "Gestión y Tecnología de los residuos sólidos y líquidos: La experiencia de Austria y Perú - Tema: Gestión de los residuos domésticos e industriales en el Perú. CEPIS"

Silva, J. 2008. "Propuesta Para el Manejo de Residuos Sólidos en la Fábrica de Cubiertos ASA S.A.C" Trabajo de investigación no Experimental para titulación. Universidad Nacional Agraria La Molina.

Paraguassú, F. y Rojas C. 2002. Indicadores para el Gerenciamiento del Servicio de Limpieza Pública. Segunda edición. CEPIS. Perú.

Pfeffer, J. 1992. Solid waste management engineering. McGraw-Hill. Canadá.

Quesada, H; Salas, J. y Romero, L. 2007. Manejo de desechos industriales peligrosos. Tecnología en Marcha. Vol. 20-2.

Sakurai, K. 1981. Análisis de residuos sólidos: Manual de Instrucción. Programa Regional OPS/EHP/CEPIS de Mejoramiento de la Recolección, Transporte y Disposición Final de Residuos Sólidos.

Ramírez, J. 1990. Manual de Sistemas y Alternativas para el Manejo de Residuos Sólidos. Honduras.

NTP 900.058.2005. 2005. Gestión de residuos. Código de colores para los dispositivos de almacenamiento de residuos. NTP 900.058.2005. 